(c) 2010 IEEE. Personal use of this material is permitted. Permission from IEEE must be obtained for all other uses, in any current or future media, including reprinting/republishing this material for advertising or promotional purposes, creating new collective works, for resale or redistribution to servers or lists, or reuse of any copyrighted component of this work in other works. 


\title{
Ecological Solutions For The Blind
}

\author{
David J. Calder \\ Curtin University of Technology \\ Bentley, Perth, \\ West Australia \\ Tel. 61-8-9266 2875
}

david.calder@cbs.curtin.edu.au

\begin{abstract}
A number of inappropriate navigation devices have been foisted onto the visually impaired population over the past twenty years. These portable electronic devices are usually mounted on a long cane adaptation, or hand-held by the visually impaired user. Their function is primarily to help prevent a collision with an obstacle in the immediate path. Some of these navigation devices use laser technology to gauge subject-to-object distance. Others use infrared transceivers or ultrasonic pulse-echo techniques to locate and warn of obstacles. Some extend their role beyond giving warning signals, to crudely assisting the user map the immediate path ahead. These devices exhibit a number of problems, the most significant of which are related to the interface display that conveys navigation/obstacle warning information to the blind user. As sound is often used in these displays, the vital auditory channel is typically compromised. The author has produced a solution, which is at an early stage of development. Several successful prototypes using tactile displays, have been produced.
\end{abstract}

Keywords: Assistive technology, ambient sound cues, disabled user, infrared proximeter, visually impaired, laser rangefinder, long cane, portable electronic device, sensory channels, sound displays, tactile displays, ultrasonic pulseecho.

\section{INTRODUCTION}

A mobility aid for the visually impaired is a portable electronic device that is either hand-held or worn by the client, which warns of obstacles ahead. These devices suffer from a number of problems, the most important of which are related to the interface that conveys information to the user. Although some use simple vibrating buttons, most aids create binary sound alerts to warn of upcoming obstacles; a method which is only capable of conveying very crude information regarding direction and proximity to the nearest object. Some of the more sophisticated devices use a very complex and confusing (to many users) audio interface in order to deliver more detailed information. But this approach often compromises the user's hearing, a critical impairment for a blind user. The author has produced an original design and working prototype which is a major first step in addressing these faults in current production solutions for the blind.

In parallel with this mobility aid development work is the requirement for the drafting of an original set of unique Digital Ecosystem guidelines, which will allow for the confident launch of this and other spin-out assistive device companies, by having mutual support across various disability modalities.

Many people have one or more progressive disabilities. These clients are dynamically interactive within an ecology that is itself constantly adapting and changing in real time. Far from being static, complex human systems are constantly interacting with one another as well as other machine systems within the ecology. As these human systems evolve, so the whole surrounding ecology evolves too.

A technology-centred approach that produces an inherently inflexible solution will be flawed. Many designs based on requirement specifications for disabled users fall short as ongoing adaptive solutions. In practice, it is currently almost impossible to offer an alternative or augmentative technology that will match and then continue to match the user requirements of an ever evolving system, as is the case where human input and output channels and the complexities of human-to-human and human-to-machine communication are concerned.

The rejection rate of currently produced assistive devices reinforces this claim and is discussed later in the paper. Each individual is a moving target for the system designer, as the day-to-day progression rate for each user is different. The long-term user requirements are therefore dynamic in essence.

There are also factors such as the time and financial investment involved in designing and manufacturing for one person in a bespoke tailor-made fashion. The answer is often a compromise.

These challenges are compounded if the user has multiple disabilities [1]. The design team, who may be focusing exclusively on one area of disability, does not always consider these multi-facetted demands. 


\section{DEVICE ACCURACY}

Many currently available orientation devices suffer from lack of accuracy. They often have a limited means of 'mapping' the terrain ahead, and even if they could, they are incapable of adequately transmitting that information to the user. Although many mobility aids can warn of obstacles up to six metres ahead and crudely convey the distance of said objects to the client, they cannot convey what would normally be regarded as field of view information to the user without compromising other critical sensory channels. Although complex GPS systems have had some success in addressing this limitation, they seldom warn of obstacles immediately ahead, are often unsuited for indoor use, may be extremely bulky to wear, typically are prohibitively expensive and often severely compromise the natural function of the auditory sense. They cannot be regarded as stand-alone systems.

If the client is presented with limited orientation feedback, not only is quality of life impaired, but also mobility may be reduced to an isolated step by step cane assisted progression typically punctuated by crude non specific on/off warning signals from a mobility aid. Relatively few visually impaired people accept the devices that are currently available. This is not surprising as the performance of these devices, for the reasons discussed above, cannot justify the hefty price tag. They will accept the Long Cane for its simplicity and predictability and the fact that it approximately a fiftieth of the cost of a sophisticated electronic aid.

\section{COMMERCIALLY AVAILABLE EXAMPLES}

There are a number of reviews such as those listed in Currently Available Electronic Travel Aids for the Blind [2]. None of these can be regarded as more than a rough guide. Clear evidence of why current aids are rejected can be found in relevant conference and journal papers such as $[3,4,5]$. Blasch for example, states that few are regularly used. Davies in 2006 refers to only limited continued use of the device [4].

Electronic Travel Aid (ETA) rejection was highlighted in a report from National Research Council [6]. This report refers to auditory interfaces that compromise the natural feedback derived from tapping a long cane. These auditory displays are still the most common interface in more sophisticated orientation devices.

The Miniguide is a small handheld device that uses ultrasonic pulses to echo locate obstacles in its path. It has the advantage of a low current requirement. However, when used indoors, most of the ultrasonic devices pick up unwanted ambient echos from adjacent walls, ceilings and surfaces which may corrupt the result. Vibration displays usually draw more current than the rest of the circuit.

The Teletact 2 uses both laser and infra red transceivers, in order to overcome some of the problems associated with each method used in isolation. An earlier version exploited a laser only, the reflected beam of which can result in a confused signal from plate glass, such as in a door or front to a building. There was also a problem with lasers picking up black objects, such as black cars or other vehicles. Grass at the side of a path could also be confusing to a laser-based system. Infra-red systems usually work well indoors, but can be adversely affected by interference from the environment, such as sunlight.

In case of both infra red and laser telemetric detection, the system transmits telemeter information. When it senses the infra red transceiver signal only, it sends a "window warning" cue to the user, in order to warn them that they may be approaching a window. The infra red system works within a range of 3 meters, and gives a window pane / black car detection up to two meters.

It uses vibrating devices located under the user's fingers. Experiments were conducted with two, four and eight vibrating devices, and the four-device solution turned out to be the most successful. The principle of this method is simple. Each finger (except the thumb) is in contact with one and only one vibrating pad. Each vibrating pad corresponds to a distance interval. If an obstacle is detected within one of the four distance intervals, then the corresponding vibrating device is activated.

Although the Teletact 2 overcomes some of the problems associated with the previous model, it is still only a go/no go device.

\section{FUNCTIONALITY OF DEVICE USER INTERFACES}

ETA devices can be broken down into two categories. First are the simpler types that warn of an obstacle in the forward vicinity of the user, but convey little or no detail with respect to position or object identification. They may use buzzers, simple warning vibration or synthetic tones as the user interface. They seldom warn of drop-offs, such as potholes, in any truly reliable way.

The second category may have enhanced range and precision, as in the case of some laser based types, but often with a far too simplistic binary information go/no go user interface, or, alternatively, use complex sonar techniques that convert ultrasonic reflected signals into a synthetic but inhuman audio signal that is presented to the user. Such devices require substantial learning and compromise the natural sound cues that are absolutely essential for a blind person.

Many of the competing products have poor and inappropriate human-machine interfaces. A recent paper in the Proceedings of the 2005 IEEE Engineering in Medicine and Biology Conference reinforces these views [7]. Velazquez et al confirm that although many ETAs have been proposed to improve mobility and safety navigation independence for the visually impaired, none of these devices is widely used and user acceptance is low.

\section{SOUND SUBSTITUTION DISPLAYS}

There are certain complex systems that attempt to emulate a bats navigational sonar strategy. However, because the bat's echo-location system relies on a sound frequency far higher than the upper limit of a human hearing, some electronic means has to be used to either, lower the pitch relative to the original ultrasonic signal or, to translate the received echo into a symbolic form that can be learned. Such a form could be a musical sequence. 
The former method benefits from a direct un-coded audio delivery signal to the user, albeit shifted down relatively in frequency. Scanned objects normally produce multiple echoes, translated by the receiver into unique invariant 'tone-complex' sounds, which users listen to and learn to recognize. In theory, the human brain should be very adept at learning and remembering certain soundsignature sequences in a similar way that it learns a musical tune. The sound signatures vary according to how far away the device is from the object, thus indicating distance. The user listens to these sounds through miniature earphones and can detect the differences between sound sequences thus identifying the different objects. This allows limited mapping and orientation for the user at a price. The steep learning curve often proves to be the point of rejection for many users, with respect to both direct and coded methods.

Any auditory user interface has the potential to interfere with the users' hearing of natural ambient sound cues. This is a critical factor for a blind user. If used in a safe environment by a truly driven person prepared to learn over time, sound signatures representing a visual scene could significantly enhance quality of life. However, the 'real world' is not safe, and there are serious safety concerns about restricting the hearing of a blind user in an uncontrolled environment.

Beyond the safety aspect, blind users have learned to depend on their hearing, and any product which continuously interferes with it may lead to a compromised alternative human sensory input. Supporting evidence for this claim can be universally found from different disciplines. Some of these have already been referenced in the preceding sections. A more specific reference can be found in Johnson and Higgins, who refer to visual auditory substitution taxing a sensory modality that is already extensively used for communication and localization [8].

\section{TECHNOLOGY DRIVEN DESIGNS}

It is now accepted by Human System Interaction (HSI) specialists, that earlier user interface designs were often driven primarily by technology rather than being user centred. Although challenging interface designs [9] require the guidelines and standards of a user centred methodology [10], the results with respect to their implementation and user acceptance are not that encouraging.

An extreme example of a technology-driven user interface design is the commonly used QWERTY keyboard layout [11]. Designed to slow input from the user in order to prevent the (mechanical) typewriter hammers from jamming during operation, the result was constrained by engineering limitations of the time.

Velazquez [7] refers to four shortcomings of existing ETAs. One of these is they provide an acoustic feedback that interferes with the blind person's ability to pick up environmental cues through hearing.

Recent studies indicate that a 20 minute usage of acoustic feedback devices causes serious human information registration, reduces the capacity to perform usual tasks and affects the individual posture and equilibrium [12]. Many audio sensory substitution devices fail because of their complex, confusing and restrictive audio feedback to the user, which blocks natural ambient sounds. They are therefore not suitable for a typical blind user who will probably have multiple disabilities. A Study by Ross and Blasch [13] clearly indicated that blind people preferred a simple tapping tactile interface to a device generated sound feedback!

\section{ECOLOGICAL SOLUTIONS}

Multiple disabilities can be physical, cognitive or both. Issues of complexity with respect to individual requirements must be seen within the context of a wider ecology of the particular user, with that person clearly at the centre, contributing to a team solution. An established and highly successful ecological approach to designing individualized education programmes for the disabled student has been refined over twenty years into a highly recommended model and is now regarded as 'best practice' [14]. This ecological approach has not as yet permeated all areas of disability support. However, the power of the digital ecosystem framework is now accepted within many other disciplines, particularly with respect to small enterprise collaboration [15].

Within small business, the advent of the web has allowed sales penetration over vast distances. Accompanying these advances have come new modes of marketing and partnership possibilities that would have been impossible only a few years ago. With this connectivity has come a fertile and dynamic business theatre that cannot be avoided if small enterprises are to survive. This interaction has led to collaborative workflow models [16].

The logic behind collaborative workflows is to produce a sequence of activities that not only produce a meaningful result, but also to facilitate small groups working together to achieve common goals. The actual physical distance and associated limitations between these entities then becomes less important as web based tools are used to link enterprises and their common aspirations [17]. The entities themselves may be small companies competing against large predator corporations, or widely dispersed cottage industries (such as those associated with assistive devices) with a common interest [18].

Beyond the standard empowerment the digital ecosystem model has provided, are more specific areas that are pertinent to such groups operating in harmony. One of the most important of these is trust evaluation [19]. Other typical support areas are logistics and privacy [20, 21].

When any one of the major human system input channels is affected, the functional potential of the whole person will be compromised. A loss of vision in particular, presents extraordinary challenges. With time, other viable modalities have been shown to compensate and become enhanced in sensitivity. This enhancement 
occurs in the compensating areas of the brain [22]. With time, the human system adapts and compensates for the loss of a modality, to some extent at least. For the assistive device engineer, however, there is a continually moving target - the requirement specification is therefore constantly changing and never fixed. Recent advances in the understanding of brain plasticity reinforce this perspective. It has been demonstrated that driving the brain with demanding sensory, cognitive and motor activities on a frequent basis will often result in a positive outcome. The reverse is also true [23].

Whereas vision impairment may often be linked to the input channel that results in sight, physical disability may also be associated with the output channels from the human system. The loss of motor speech is often associated with paraplegia or quadriplegia. Communication and mobility may be simultaneously compromised.

It is generally accepted by interventionists that an early programme must provide the learner of communication skills with a systematic means of restoring and maintaining control over his or her environment. Augmentative and alternative communication specialists have for many years, made use of an ecological model to promote control for the disabled learner of communication skills [24]. This model should also be applied to support systems for the blind.

\section{AUTHOR'S PROTOTYPE}

The Innovation in the authors own first prototype design (See Figure 1) is summarised as follows:

- A portable mobility aid incorporating warning obstacle ahead information with mapping capabilities as a stand-alone device. It does not rely on GPS or other external signals such as required by radio tags.

- The system may also be stand-alone in another sense, as it can in some circumstances, replace a standard long cane or guide dog or third person assistance. In such a configuration it is therefore a hands-free device! Dependent on specific user requirements, the system can also be configured to be an augmentative assistive device to be used with a standard cane or dog. This would be a typical urban setting.

- A unique tactile display is used to convey system output data (field of view features) to the user.

a A more advanced design is currently also under development. It is unique in that it offers environmentally contextual drop-off and step up warning in a hands-free design. Of all the competition, only the Laser Cane offers drop-off warning, but it is not hand-free.

Experiments have been carried out using ultrasonic transceivers as part of a range-finding system, in order to produce the first prototype. There is a tactile interface which has been adapted to use on the trunk of the user's body in the hands-free mode, in the handle of a modified long cane in augmentative mode and against the palm of the hand in the alternative stand-alone configuration. The casing, controls and displays vary depending on these adaptations. Only in extreme warning situations where an object is suddenly detected within two meters across the path of the user, will an audible sound compliment the signal from the tactors. In this way, the user audition cues are not compromised except in an emergency situation.

Sub-sonic vibrations commence at the lowest frequency from a distance of approximately 6 metres in a cone of sensitivity extending from the system in a straight ahead aspect, at approximately 60 degrees. Cone sensitivity is greatest at the center axis. Echo sensitivity about the axis takes the form of a bell shaped curve, strongest at the center line.

Of all the alternatives, this method allows for a small power requirement and an alkaline PP9 battery is able to drive the front end transceivers as well as the tactile interface display for several hours. This facilitates portability and weight in a system which needs to be as unobtrusive as possible.

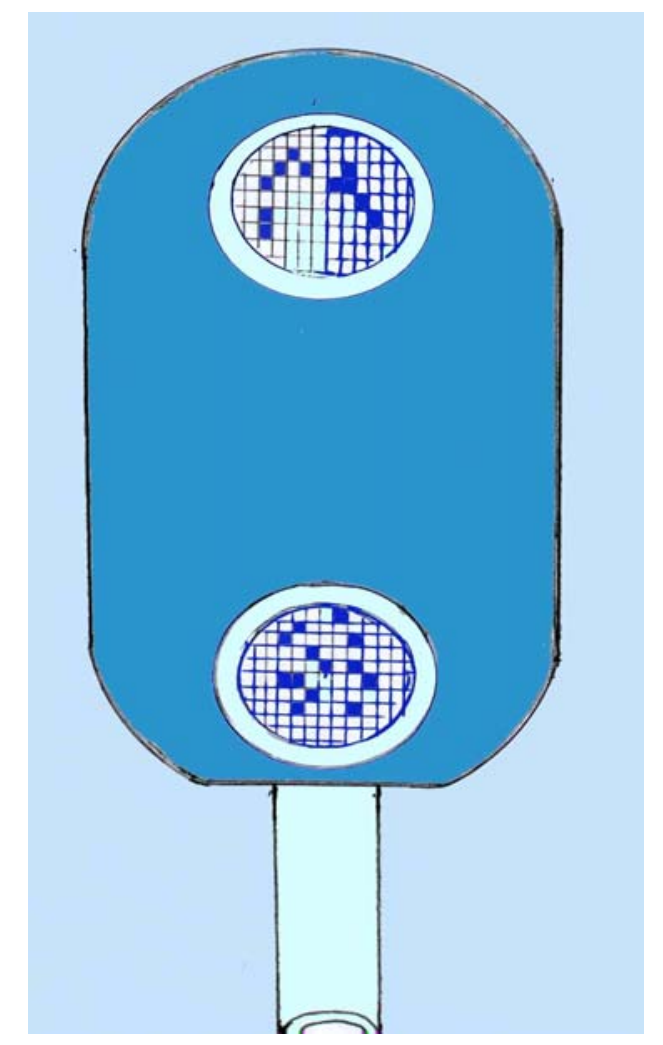

Fig 1. Front view of the first prototype ultrasound system as fitted to a standard long cane. Note this first prototype is not hands-free, although later versions are.

Initial tests have indicated the system works extremely well at detecting objects in the path of a user in an outdoor setting. However, like most similar systems, it suffers from some echo signal interference in an indoor setting. It 
also cannot be relied upon to indicate sudden surface anomalies and undulations.

This first prototype would best be used as an augmentative device where a cane or dog may be required for close up detection and drop offs, such as steps down or potholes in the road surface.

Subsequent designs are currently being constructed that address drop-off and indoor situations, although the simplicity of the original specification will be sacrificed.

The main reason for the original design was to test the novel tactor display interface. This has proved to be successful in this first design and has resulted in further funding for a more advanced set-up which will be trialed after the system has been miniaturized. At this juncture, the system has been successfully tested by a small sample of visually impaired people. User feedback is ongoing and has been valuable and constructive at this current juncture. The typical first system test configuration has been the simpler cane mounted version (See figure two).

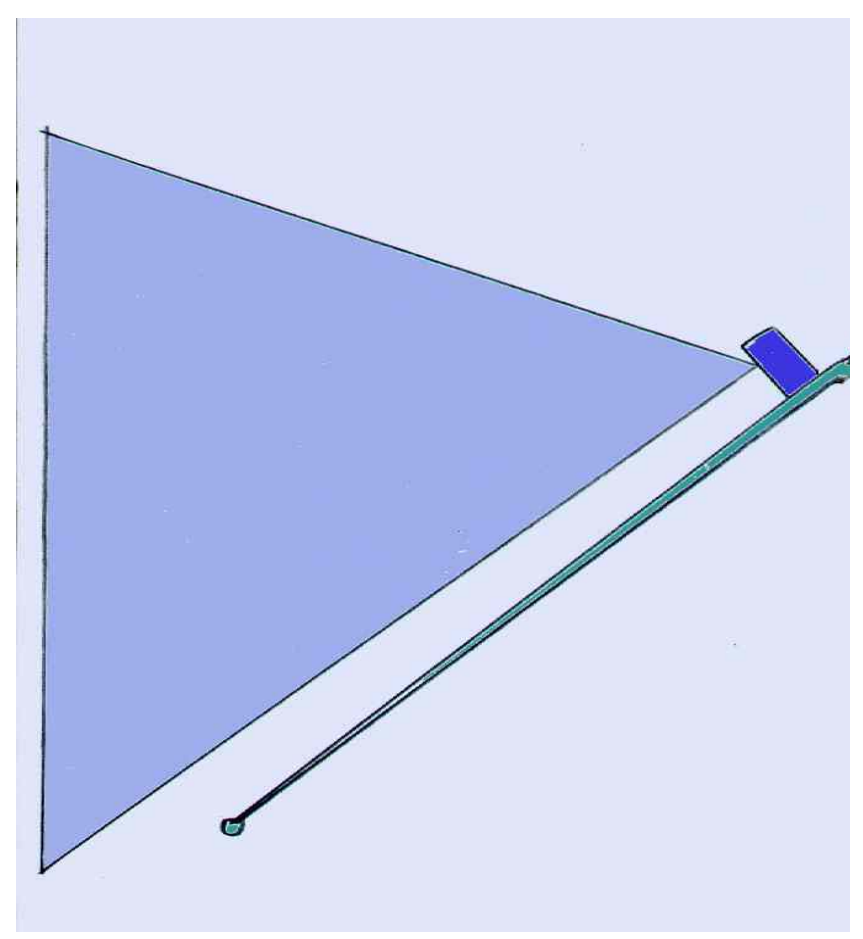

Fig. 2 The side view first prototype ultrasound system mounted on a cane and showing the cone of sensitivity radiating out in front of the user. Unlike IR and laser beams with narrow profiles, this system provides relatively wide area coverage. Power consumption is relatively low too.

It is not possible to expand upon the specifications of this particular interface at this point in time due IP sensitivity. However, there has been an interest from commercial investors and specialists. It may be possible, it is hoped, to soon expand on these specification details in future papers.

\section{CONCLUSION}

There has been considerable resistance demonstrated to products that 'advertise' a user's disability. Unlike many commercial devices, an ideal system should be configurable for both blind and deaf/blind people and be intuitive in its human requirement.

Although this has yet to be fully quantified, it has been estimated by the author that a viable and competitive device could be produced for under $\$ 300$ from standard off-shelf components. The cost of current products is still too high.

As most current devices are produced by small, unlisted companies, there is little in the way of publicly available, reliable sales figures, and as such the addressable market is not well defined. However, interviews conducted with industry experts, in addition to the small size of the companies themselves, suggest that these competing devices have so far failed to achieve any significant market presence. Burns states that a device should be picked up and used immediately. It is often the tedious learning procedures amongst the other human factors issues listed in this paper, that are often the downfall of these devices [25].

The aim should be to retain as far as possible, those learned schemas that the user is comfortable with, but at the same time extend the possibilities of range and resolution by using the latest appropriate technology. Taking the users background experience into account should be one of the major considerations of a good design; a characteristic that is sometimes neglected in current products.

The author has attempted to produce solutions to the many problems associated with assistive device rejection, particularly for the blind and the all important user display in particular.

Firstly, an ecological approach will be used in the development of a range of navigation tools for the blind. The failings of commercially available mobility aid displays have, and will continue to be, the basis for design change by our team. Devices will be produced that are intuitive to use and do not require a steep learning curve. Other sensory modalities, particularing hearing will not be compromised by future designs. The range will permit an affordable and robust device alternative, that can be produced cheaply for third world requirements. This is not being done at the present time!

\section{REFERENCES}

[1] J. Jacko, H. Bautsch, “A Conceptual Framework For The Integration of Clinical Diagnosis, Functional Capabilities and Classes of Technology”, University of Wisconsin Lab for HumanComputer Interaction. Technical Report series no. 1131, 2000, pp. $1-59$

[1] Y. Duen, "Currently Available Electronic travel Aids For The Blind” 2007. [Online] www.noogenesis.com.eta/current.html

[2] B. Blasch, results of A National Survey of Electronic Travel Aid Use. Journal of Visual Impairment and Blindness 83, pp 449-453. 1999.

[3] T. Davies, C. Burns and S Pinder, "Using Ecological Interface Design to Develop an Auditory Interface for Visually Impaired travelers,” Proc. Of OZCHI 2006, Sydney, Australia. 2006

[4] K Young-Jip, K. Chong-Hui and K. Byung-Kook, “ Design of Auditory Guidance System For The Blind With Signal 
Transformation from Stereo Ultrasonic to Binaural Sound,” Proc of $32^{\text {nd }}$ ISR (International symposium on Robotics), April 2001.

[5] Committee on Vision "Electronic Travel Aids: New Directions For research," Working group on Mobility Aids For The Blind, National research Council, pp 74, National Academy press, Washington, DC 1986.

[6] Velazquez, E. Pissaloux and F. Maingreaud, "Walking Using Touch”, Proc. Of 2005 IEEE Engineering in Medicine and Biology $27^{\text {th }}$ Annual Conference, Shanghai, China, 2005.

[7] L Johnson and C Higgins, "A Navigation Aid for the Blind using Tactile-Visual Sensory Substitution,” Department of Electrical and Computer engineering program, university of Arizona, Tucson USA. 2006.

[9] A. Edwards, Extra-ordinary Human-Computer Interaction: Interfaces for Users with Disabilities, Cambridge University Press, 1995.

[10] A.Dix, J Findlay, G. Abowd, R. Beale, Human-Computer Interaction, New York: Prentice Hall, pp. 160 - 173.

[11] B. Shneiderman, Designing The User Interface, Reading MA: Addison Wesley Longman, 1998, pp. 308 - 311.

[12] J. Hakkinen, "Postural Stability and Sickness symptoms After HMD Use”, Proc. Of IEEE International conference on Systems, Man and cybernetics, Hammamet, Tunisia, 2002

[13] D. Ross and B. Blasch, "Wearable Interfaces for Orientation and Wayfinding,” Proc. Of ASSETS 2000, Arlington, Virginia, USA. 2000

[14] B. Rainforth, J. York, C. Macdonald, Collaborative Teams for Students With Severe Disabilities, Baltimore: Paul Brookes, 1993, pp. 71- 83.

[15] E. Chang, M. West. "Digital Ecosystems and Comparison to Collaboration Environment”. WSEAS Transactions on Environment and development 2, 2006, pp. 1396-1404

[16] L. Pudhota, Chang E, "Modelling the Dynamic Relationships between Workflow Components” ICEISI Porto, Portugal, 2004.

[17] D. Neumann," An Introduction to Web Objects". On-line at http://mactech.com/articles/mactech/Vol.13/13.05/WebObjects Overview. 2004

[18] M. UlieruR. Brennan Scott, “The Holonic enterprise: a model for Internet-enabled Global Manufacturing Supply Chain and workflow Management", Canada, 2000

[19] E. Chang, T. Dillon and F Hussain, "Trust and Reputation for service-oriented Environments: Technology for building Business intelligence and Consumer Confidence,” John Wiley and Sons, West Sussex, England, 2006

[20] M. Clark, P. Fletcher et al. Web Services Business Strategies and Architectures. Expert press. 2002

[21] G. Skinner and E. Chang. "A Projection of the Future Effects of Quantum Computation on Information Privacy and Information Security” International Journal of Computer Science and Network Security 6, 2006, pp166-172"

[22] E. Goldstein, Sensation and Perception, Boston: Brooks Cole, 1999, pp. $377-378$

[23] M. Woodlee, T. Schallert. "The interplay between behavior and neurodegeneration in rat models of Parkinson's disease and stroke”, Restor Neurol Neurosi, 2004, 22(3-5); pp 153 - 161

[24] J. Reichle, J. York, T. Sigafoos, Implementing Augmentative and Alternative Communication - Strategies for Learners with Severe Disabilities, Baltimore: Paul Brookes, 1991, pp. 61 - 70.

[25] C. Burns and J Hajdukiewiez, "Ecological Interface Design," Florida: CRC Press, pp 4, 2004. 